\title{
Huddling behavior of spiny mouse pups toward foster siblings from another species
}

\author{
JOSEPH MIELE \\ State University of New York, Albany, New York \\ JENNIFER WHEELER MAKIN \\ George Peabody College of Vanderbilt University, Nashville, Tennessee \\ and
SIMONE RUSSO, KATHLEEN CAMERON, FRANK COSTANTINI, and RICHARD DENI
Rider College, Lawrenceville, New Jersey

\begin{abstract}
In Experiment 1, individual newborn spiny mouse pups were cross-fostered to Norway rat litters. Each rat dam was housed with six of its own pups and a foster spiny pup. A 30-min observation was videotaped on Day 5 postpartum. The dam was removed, and huddling contacts made by the spiny pup with its foster siblings were recorded in a situation in which all the animals could move freely. The total time spent huddling and the conditional probability of moving from being out of contact to being in contact were recorded for two contact categories: same-sex rat pup(s) and opposite-sex rat pup(s). During the observation period, spiny mice spent significantly more time in huddling contact with individual or grouped foster sibling rats of the same sex. Experiment 2 tested similar questions, but with different methods. All fostering and rearing procedures were the same as those used in Experiment 1; however, huddling tests took place in an apparatus in which discrete choices between two alternative huddlemate categories were recorded. Spiny mice were tested at 5 and 10 days of age, with ratsibling agemates as huddling stimuli. In a choice test between two male or two female rat siblings, male spiny mice huddled more with same-sex rat pups, but female spiny mice showed a marked avoidance of tactile contact with opposite-sex rat pups. The results were discussed in terms of gender differences in companion discriminability and chemical cue production.
\end{abstract}

The study of attachment behavior has often taken the form of exposing developing animals to artificial animate and inanimate stimuli in an attempt to alter the normal course of behavioral development. Certain of these studies have focused on the differential sensitivity of adults of both sexes to the effects of artificial stimuli used in the selection of a social companion from among various categories.

In an early study of this type, Mainardi, Marsan, and Pasquali (1965) altered the odor of mouse dams (Mus musculus, SWM/Mai) with perfume and thus exposed the developing pups to a constant artificial stimulus cue for identification. In choice tests carried out on the offspring at adulthood, Mainardi et al. found that only females reared with the artificial odor selected perfumed companions over those with an unaltered scent. Males showed no selection preference. Female mice

We thank Donna Reisert, Susan Wilson, Rene Piper, and Lisa Budzek for their assistance. Address reprint requests to Richard Deni, Psychology Department, Rider College, Lawrenceville, New Jersey 08648. were more discriminating in their selections of adult companions.

A method for altering animate stimuli during development has been the cross-fostering technique. Quadagno and Banks (1970) reported gender differences in the selection of social companions by adult mice reared with continuous exposure to altered animate cues. Two rodent species were used in that study, and species $\mathrm{x}$ gender interactions were found. Wild pygmy mice (Baiomys taylori ater) were compared with inbred house mice (Mus musculus, C57BR/cdJ) after reciprocal cross-fostering. When tested as adults, pygmy mouse females discriminated the foster species from their own and consistently huddled with companions from the foster species. Male pygmy mice showed no species preference. When house mice were tested, both males and females preferred companions from the foster species.

Aspects of gender-based social discrimination have been observed following brief exposures to alien stimuli at the time of preference testing. Deni, Miele, Wilson, Longo, Miernicki, and Silvers (1982) recorded the huddling and touching of 5- to 7-day-old Norway rat 
pups (Rattus norvegicus, Long-Evans) when different categories of companion pups were available: same- and opposite-sex siblings, and same- and opposite-sex strange agemate rat pups. Deni et al. (1982) found that both male and female rat pups, when tested on clean cage bedding or on bedding soiled by a strange dam and litter, maintained significantly more contact with opposite-sex siblings over all other categories. However, when tested on soiled bedding from the home cage, both male and female pups spent more time in contact with same-sex siblings. Conspecific and sex-specific odors are implicated as determinants of attachment responses, and interactions among various sources of companion odor stimuli must be expected.

Cues from strange conspecifics and strange bedding soiled by conspecifics are less intense than those present during exposure to a live stimulus animal from an alien species. Live alien stimuli have had different effects depending upon the gender of the animals involved. Deni, Wilson, and Reisert (1983) tested precocial spiny mouse pups (Acomys cahirinus) in an approach-choice situation that required selection of companion animals from among several categories, including same-sex versus opposite-sex conspecific and alien (Norway rat) pups and live versus dead alien pups. Reliable preference patterns were obtained during a single brief choice test. Newborn female spiny mice preferred to remain near opposite-sex rather than same-sex alien newborn rat pups. Male spiny mice showed no differentiation. Newborn female spiny mice showed the same test results when dead newborn rats were used as selection stimuli. Gender-based choice preferences were not found, however, when strange newborn conspecifics were used as selection stimuli, which suggests that the effect was exclusively interspecific. Gender differences in exploratory or even predatory behavior may be involved in regulating approach and proximity responses toward stimulus animals.

The above techniques have stimulated research on the environmental determinants of social responsiveness and attachment behavior. The studies of gender-based attachment behavior reported here used cross-fostering to manipulate a complex of alien cues provided by foster siblings from a different species.

\section{GENERAL METHOD}

\section{Subjects}

The subjects were infant spiny mice (Acomys cahirinus) bJrn to multiparous parental pairs. Only a single mouse pup was selected, at random, from a given litter. The parents were housed together in standard plastic breeding cages, with food and water always available. Colony rooms were illuminated between 0630 and $1830 \mathrm{~h}$ each day.

\section{Cross-Fostering Procedure}

Newborn spiny mice were removed from the parental cage on Day 1 and fostered into specially prepared mother-litter cages containing Norway rats (Rattus norvegicus, Long-Evans).
Primiparous rat dams were housed with their litters in standard plastic cages that contained pine bedding material; food and water were always available. On Day 1 postpartum, recipient litters randomly were reduced to three male and three female pups. Clean plastic containers and glass beakers, rather than direct handling, were used for transferring the animals.

\section{EXPERIMENT 1}

In this study, huddling by fostered spiny mice toward rat siblings was recorded in a situation in which all animals were free to move. The gender of the spiny pups used as the subjects was a between-subjects factor. The classification of rat siblings huddled against (samesex vs. opposite sex) was a within-subject factor. The objective was to determine if huddling and touching behaviors by spiny mice would vary as a function of gender, as had been suggested by previous reports.

\section{Method}

Subjects. The subjects were four male and four female spiny mice. On Day 1, each spiny pup was cross-fostered and remained housed with a rat dam and her reduced litter for the next 6 days.

Apparatus. A separate plastic cage bottom, identical to that used in the home cage, was used for all observations. A thin layer of clean pipe shavings was used as bedding. Videotaping, done in a separate small room, required a camera positioned $1.5 \mathrm{~m}$ above the open observation cage. Overhead fluorescent light sources were used for illumination, and the room was maintained at $70^{\circ}-73^{\circ} \mathrm{F}$. Videotapes were coded in two ways. Pushbutton timers able to resolve events to the nearest $.1 \mathrm{sec}$ were used to record duration of huddling contacts by category of sibling. A stream-of-behavior checksheet was also used to record the sequence of out-of-contact and in-contact transitions produced by each subject.

Procedure. On Day 5, each spiny mouse subject and its foster siblings were transferred into the observation cage and taken to the test room. Observations were conducted between 1300 and $1500 \mathrm{~h}$ and lasted $30 \mathrm{~min}$. Before being transferred, each rat pup was marked for identification with water-based white typing correction fluid applied to the animal's dorsal surface. The observers were blind to the gender of subjects and huddlemates. During playback of tapes, each session was coded by recording the duration of two classifications of physical contact between the spiny subject and its rat foster siblings: contact with only same-sex siblings and contact with only opposite-sex siblings.

\section{Results and Discussion}

Intermittent reliability checks were made, and agreement between any pair of observers was always above $90 \%$. The dependent measure of duration of physical contact with foster siblings was obtained twice: once for contacts with same-sex rat pups and again for contacts with opposite-sex rat pups. These scores were used as raw data for a two-factor mixed analysis of variance (Kirk, 1968, p. 248). The analysis resulted in a significant main effect for sex of rat sibling huddled against. Significantly more contact occurred with samesex siblings $[F(1,6)=7.47, p<.05]$. Means $(M s)$ and standard deviations (SDs) for contact durations (in seconds) were as follows: same-sex huddlemates, $\mathbf{M}=$ 
120.3, $\mathrm{SD}=79.7$; opposite-sex huddlemates, $\mathrm{M}=42.1$, $\mathrm{SD}=28.0$. No other $\mathrm{F}$ ratios were significant. The stream-of-behavior data were used to compute transition probabilities between out-of-contact and two other states: in contact with same-sex and with opposite-sex foster siblings. Trends similar to the duration measures were obtained without significant effects. For comparison, the mean transition probabilities for the two classifications were: for male spinys contacting same-sex huddlemates, $\mathbf{M}=.36$, and opposite-sex huddlemates, $\mathbf{M}=.28$; for female spinys contacting same-sex huddlemates, $\mathrm{M}=.57$, and opposite-sex huddlemates, $\mathrm{M}=.32$.

Previous research on spiny pups huddling with strange rat neonates also showed that the gender of the spiny mouse predicted the choice of huddlemate (Deni et al., 1983). However, the treatment effects in that case were much more complex than those found here. For example, Deni et al. (1983) found that newborn female spiny mice tended to huddle more with opposite-sex strange rat neonates than with same-sex animals. In addition, female spiny mice, first tested at 10 days of age, huddled more with same-sex strange rat pups. Male spiny mice, tested as newborns or as 10-day-olds, seemed to show no sex differentiation. Perhaps the foster-rearing experiences involved in the present study served to reduce the complexity of any hypothetical sex-differentiation capacity. The difference between the results of Experiment 1 and those of previous research with strange-rat huddle stimuli may involve the possible different functions of the same behavior patterns. For example, exploration could explain the prior finding that spiny pups approach and touch strange neonates of a different species. This behavior may have been more highly variable and subject to age and gender interactions. In Experiment 1, the huddling behavior may have an attachment function, since the spiny pups were reared with the stimulus animals. The stability of filial attachment bonds to an alien species might involve regulation by a main effect such as that obtained in Experiment 1.

\section{EXPERIMENT 2}

Again, spiny mice were cross-fostered into rat litters, and their huddling behavior with foster siblings was observed. The test conditions involved a two-option choice situation with either same-sex or opposite-sex foster siblings positioned separately on opposite sides of a barrier. Selection of a site was correlated with approach toward stimulus animals of a particular gender.

\section{Method}

Subjects. Four male and four female spiny mice were selected as subjects. Cross-fostering procedures and selection and preparation of recipient rat litters were the same as in Experiment 1. For the next 11 days, each spiny pup remained housed with a rat dam and her reduced litter.

Apparatus. The animals were observed inside a tall plastic container with floor dimensions of $33 \times 15 \mathrm{~cm}$. The floor was covered with a thin layer of clean pine bedding and divided into two equal $12 \times 13 \mathrm{~cm}$ zones by a wire-cloth "bump" placed in the center and flush with the two longer walls of the compartment. This obstruction separated the two groups of rat pups used as huddling stimuli. However, spiny pups could climb over the obstacle and move freely between the two zones. All other conditions of testing and apparatus were the same as in Experiment 1 .

Procedure. The subjects were videotaped on Days 5 and 10 . Prior to each observation, the rat dam was removed from the home cage, and two male and two female rat pups were selected at random for transfer to the test enclosure. The rat pups were grouped in like-sexed pairs. Each pair was placed randomly into a zone of the test enclosure, which was then placed under the videocamera. The spiny mouse subject was then placed on the centerline wire-mesh "bump." A 30-min videotaped observation was then carried out. The observers were kept blind to the gender of the rat pup stimulus pairs through the use of a coding system that did not involve gender-identification labels. This system did not require marking the animals. Only the zone in which each gender pair was positioned was labeled. During playback of tapes, the total amount of time the spiny pup spent in each zone was recorded. Zones contained either two same-sex or two opposite-sex foster rat siblings. Recording was divided into the time spent touching and not touching a stimulus pup in the zone. Within-subjects classifications were: zone (same-sex vs. opposite-sex foster rat sibling) and physical proximity (touching vs. not touching).

\section{Results and Discussion}

Intermittent reliability checks produced agreement scores always above $95 \%$. The Day 5 and Day 10 scores were averaged to provide, for each subject, four raw scores analyzed for group differences with a three-factor mixed analysis of variance (Kirk, 1968, p. 298). Only two significant $F$ ratios were obtained. The first of these was a significant interaction effect between the sex of stimulus rat pup (same sex vs. opposite sex) and proximity (touching vs. not touching) $[F(1,6)=12.18$, $\mathrm{p}<.05]$. Newman-Keuls test results indicated that spiny mice observed in the zone containing same-sex rat siblings spent significant $(\mathrm{p}<.05)$ more time touching $(M=300.7, S D=200.2)$ than not touching $(M=$ $119.1, \mathrm{SD}=155.2)$ those stimulus pups. The same comparison for the opposite-sex zone showed a nonsignificant but opposite trend (touching: $M=157.4, S D=$ 180.3; not touching: $M=201.1, S D=239.1$ ). The tendency for foster-reared spiny mice to come into physical contact with their rat siblings was affected by sibling gender. Compared with the data from Experiment 1 , the present data suggest that a higher resolution was obtained in the measurement of a hypothetical sex-differentiation mechanism. The Experiment 2 data also support the idea that spiny pups attach more strongly to same-sex huddlemates. Opposite-sex siblings produced more avoidance of physical touching.

A significant three-way interaction was also obtained $[F(1,6)=7.53, p<.05]$. The means and standard deviations for the duration scores in this interaction appear in Table 1. Only one specific comparison was found to be significant ( $p<.05$ ) according to the Newman-Keuls test. Female spiny mice in the zone 
Table 1

Means and Standard Deviations for Time (in Seconds) Spent by Spiny Mice Touching or Not Touching Foster Rat Siblings

\begin{tabular}{llll}
\hline \multicolumn{1}{c}{ Gender } & Proximity & Mean & SD \\
\hline \multirow{4}{*}{ Same Sex } & Male Spiny Mice & & \\
& Touching & 264.7 & 184.2 \\
Opposite Sex & Not Touching & 131.4 & 213.5 \\
& Touching & 235.2 & 208.8 \\
& Not Touching & 150.2 & 210.2 \\
& Female Spiny Mice & & \\
Same Sex & Touching & 336.7 & 237 \\
& Not Touching & 106.6 & 101 \\
Opposite Sex & Touching & 79.7 & 127.2 \\
& Not Touching & 252.1 & 287 \\
\hline
\end{tabular}

containing opposite-sex foster rat siblings (the final two means displayed in Table 1) significantly avoided touching a stimulus pup. The trends toward more touching than not touching in all other contexts were stable. This finding conforms very closely to an earlier, similar study of approach behavior by spiny pups toward strange rat neonates (Deni et al., 1983). When spiny mice were tested in a two-choice situation and confronted for the first time with strange rat neonates, they tended to avoid physical proximity to the oppositesex rat pup in favor of proximity to the same-sex rat. A tentative conclusion from Experiment 2 is that female spiny mice are less likely to develop an exposurebased attachment to opposite-sex foster rat siblings, whereas they do attach to same-sex foster rat siblings.

\section{GENERAL DISCUSSION}

Previous research on huddling and companion selection by young spiny mice tested with unfamiliar alien rat pups showed that gender often correlated with proximity preferences. Deni et al. (1983) found female spiny mice to be more consistent than males in showing selection preferences for opposite-sex rat pups, whether the latter were alive or dead. The result of the two experiments reported here contrast sharply with the direction of that preference, but reinforce the importance of gender as a mediating factor. After foster rearing with alien rat pups, both male and female spiny mice had formed stronger attachments to same-sex foster siblings. Experiment 2 showed that gender could further affect attachment behavior in that, if female spiny subjects selected the site containing opposite-sex foster rat pups, they showed a significant tendency to avoid touching the rats. Males did not show this effect. Female spiny mice seemed to discriminate more than males, in a pattern similar to previous reports of companion selections by adult house mice and pygmy mice (Mainardi, Marsan, \& Pasquali, 1965; Quadagno \& Banks, 1970).

Previous research has indicated that foster rearing results in a broad spectrum of change in behavioral and physiological indexes, including reduced locomotor activity, increased arousal, slower avoidance learning, reduced aggressiveness (Paschke, Denenberg, \& Zarrow, 1971) and increased frequency of convulsive seizures (McCarty \& Southwick, 1977). Singly or in combination, these side effects could interact with the formation of attachments between the fostered animal and the alien siblings. These implications extend far beyond the present data. What is known, however, as a result of these two experiments, is that male and female fostered spiny mice functioned differently when their attachment to foster rat siblings was measured.

Maternal influences must be taken into account whenever sex differences are found. Moore and Morelli (1979) reported that rat dams lick the anogenital region of male pups more than they lick the same area of female pups. In addition, these authors found that a male foster pup elicited more licking than did a female foster pup. Specific studies must be undertaken to determine how foster rat dams react to the introduction of spiny pups. Since the spiny mouse is such a highly precocial animal, particular attention must be paid to the precise aspects of spiny-rat interaction during the early postnatal fostering, since spiny mice may differentially stimulate their rat foster siblings according to the rats' genders. Moore (1981) suggested that olfactory cues from the testicular secretions of neonatal male rats lead to differential licking of male and female pups by the dam. A similar mechanism could exist to regulate interspecies interaction, whether that interaction be exploratory, predatory, or filial.

The Experiment 2 data raise a difficult question in the case of the strong tendency for female spiny mice to avoid contact with male rat siblings when in the opposite-sex zone of the test enclosure. That finding may indicate the presence of an aversion that the Moore (1981) study suggested may be based on the early emission of chemical cues from the gonadal activity of male rat pups.

\section{REFERENCES}

Deni, R., Miele, J., Wilson, S., Longo, J., Miennicki, M., \& Silvers, M. Effect of cross-litter pup and cage bedding exchanges on huddling in rat pups. Psychological Record, 1982, 32, 543-549.

Deni, R., Wilson, S., \& Reisert, D. The approach and proximity behavior of spiny mouse pups (Acomys cahirinus) toward strange neonates: Effects of gender and species of stimulus pup. Bulletin of the Psychonomic Society, 1983, 21, 239-242.

KIRK, R. E. Experimental design: Procedures for the behavioral sciences. Belmont, Calif: Brooks/Cole, 1968.

Mainardi, D., Marsan, M., \& Pasquali, A. Causation of sexual preferences of the house mouse: The behavior of mice reared by parents whose odor was artificially altered. Societa Italiana di Scienze Naturali e del Museo Civico di Storia Naturale, 1965, 104, 325-338.

McCArty, R., \& Southwick, C. H. Effects of parental environment on the prevalence of convulsive seizures in Onychomys torridus. Developmental Psychobiology, 1977, 10, 359-364.

Moore, C. L. An olfactory basis for maternal discrimination of sex of offspring in rats (Rattus norvegicus). Animal Behaviour, 1981, 29, 383-386.

Moore, C. L., \& Morelli, G. A. Mother rats interact differently with male and female offspring. Journal of Comparative and Physiological Psychology, 1979, 93, 677-684.

Paschke, R. E., Denenberg, V. H., \& Zarrow, M. X. Mice reared with rats: An interstrain comparison of mother and "aunt" effects. Behaviour, 1971, 38, 315-331.

Quadagno, D. M., \& Banks, E. M. The effect of reciprocal cross fostering on the behaviour of two species of rodents, Mus musculus and Baiomys taylori ater. Animal Behaviour, 1970, 18, 379-390.

(Revised manuscript received for publication July 5, 1983.) 